\title{
Parameter Calibration of VISSIM Simulation Model Based on Genetic Algorithm
}

\author{
Nuerlan Muhan ${ }^{1}$, Yong Qin ${ }^{2}$, Qinghua Zhang ${ }^{3}$, Yanfang Yang ${ }^{1}$ and Zundong Zhang ${ }^{1}$ \\ ${ }^{1}$ School of Traffic and Transportation, Beijing Jiaotong University, Beijing 100044, China \\ ${ }^{2}$ State Key Laboratory of Rail Traffic Control and Safety, Beijing Jiaotong University, Beijing 100044, China \\ ${ }^{3}$ Jinhua Institute of City Planning and Design, Jinhua 321000, China
}

\begin{abstract}
Genetic algorithm is used to calibrate four driving behavior parameters and a set of calibration procedure is put forward based on VISSIM. Using the approach maximum queue length and travel time as the evaluation indexes and applying the calibration method to a single signalized intersection of Yizhuang Zone in Beijing, which validates the effectiveness and practicability of the genetic algorithm in VISSIM parameter calibration.

Index Terms - Parameter calibration, genetic algorithm, microscopic traffic simulation, single signalized intersection.
\end{abstract}

\section{Introduction}

With the development of Intelligent Transportation System in our country, the traffic simulation technology has been widely used in traffic management, traffic control and other fields. In traffic simulation model, a large number of parameters are used to describe the traffic environment, vehicle performance and driving behavior characteristics, and the values of those parameters have great influences on the result of the simulation. However, the default values of those parameters are often confirmed according to the traffic situation of the software development countries, and are not suitable for the traffic flow characteristics of our country. For a specific simulation software, we must calibrate the model parameters to ensure the accuracy of the simulation result.

Domestic and foreign scholars have studied the calibration of microscopic simulation models one after another. Benekohal ${ }^{[1]}$ was the first one to establish the framework of the calibration and verification of microscopic simulation model; Hellinga ${ }^{[2]}$ put forward the guiding principle of parameter calibration for VISSIM, but he didn't indicate the specific calibration method; $\mathrm{Cheu}^{[3]}$ was the first one to apply the genetic algorithm to the parameter calibration of microscopic simulation model and built the FRESIM model of an expressway, and then calibrated 12 parameters of traffic flow model; Park and Schneeberge ${ }^{[4]}$ came up with a parameter calibration process including 9 steps and calibrated a model building at a single intersection based on VISSIM; Park and $\mathrm{Qi}^{[5]}$ applied the genetic algorithm to the parameter calibration of VISSIM and chose average travel time as the evaluation index to calibrate eight parameters; Zhiming $\mathrm{Li}^{[6]}$ chose vehicle average delay as the evaluation index and used the genetic algorithm to calibrate seven parameters of one intersection model for Shijiazhuang city; Yang Wang ${ }^{[7]}$ chose speed and flow as the evaluation indexes, and used the orthogonal experiment to calibrate the model building at the access of urban expressway; Quan $\mathrm{Yu}^{[8]}$ and others used the orthogonal experiment to calibrate six parameters of a model building at a single intersection of Beijing South-Middle Corridor based on VISSIM. In this article, we put forward a calibration process based on the genetic algorithm and select four sensitive parameters to achieve the automatic correction. We select VISSIM as the simulation platform and apply the calibration method to a single intersection of Yizhuang Zone in Beijing, and verify the validity of this model by analyzing the errors of two evaluation indexes. We choose the maximum queue length and travel time as the evaluation indexes, and lay the foundation for analyzing the intersection quantificationally and comprehensively. We also introduce the weight coefficient to determine the relationship between maximum queue length index and travel time index, which has a certain practical significance. Let's begin with the genetic algorithm.

\section{Genetic Algorithm}

The genetic algorithm is a kind of heuristic optimization algorithm, which combine natural genetics with computer science organically with strong problem-solving ability and wide adaptability, and acquire a good effect in the field of traffic engineering. The basic idea of the genetic algorithm is that comparing the individual fitness in every generation of genetic algorithm to find out the fine individual and product a new-generation with individuals by using genetic manipulation such as genetic crossover and genetic variation. After many iterations we get the optimal individual ${ }^{[9]}$.

Basic steps for algorithm in GA are as follows ${ }^{[10]}$ :

1) Initialize a population of chromosomes.

2) Evaluate chromosomes in the population

3) Create offspring or new chromosomes by mutation and crossover from the pool.

4) Evaluate the new chromosomes by a fitness test and insert them in the population.

5) Check for stopping criteria, if satisfies return the best chromosome else continue from step 3.

6) End

After we choose the algorithm, we should confirm the calibrated parameters which have significant influences.

\section{Confirm the Calibrated Parameters}

Before calibrating the traffic model, we need to confirm the model parameters which have significant effects on the indexes of the model performance measurement, which is called parameter sensitivity analysis. The core principle of 
sensitivity analysis is that changing the value of one undetermined parameter in the case of other parameters remain unchanged. Through multiple simulation tests, we get multiple simulation output values under different levels of this undetermined parameter. Then using the single-factor analysis of variance to confirm whether the undetermined parameter has a significant influence on the evaluation indexes, and which has a significant impact would be listed as the calibrated parameter ${ }^{[7]}$. Establish a typical urban road intersection to finish the sensitivity analysis, and the geometric condition of this intersection is as follows: the east approach has a exclusive right-turn lane, a exclusive straight lane, a lane shared by left turning and straight ahead traffic and a nonmotor vehicle lane. The geometric characteristics of the west approach, the north approach and the south approach is consistent with the east approach.

In this article, eight model parameters of VISSIM including maximum look-ahead distance, average standstill distance, additive part of safety distance, multiple part of safety distance, waiting time before diffusion, minimum headway, maximum deceleration, accepted deceleration are selected for the sensitivity analysis. Taking maximum lookahead distance as an example to describe. The unit of maximum look-ahead distance is meter, and the values of this parameter are: $175,200,225,250,275,300$. The simulation output values of maximum queue length and travel time under maximum look-ahead distance's different levels in different periods (10 minutes) are as TABLE I:

TABLE I Maximum Queue Length and Travel Time under Maximum Look-Ahead Distance's Different Levels

\begin{tabular}{|c|c|c|c|c|c|c|c|}
\hline \multicolumn{2}{|c|}{$\begin{array}{ll}\text { Levels } & \text { Periods } \\
\end{array}$} & 1 & 2 & 3 & 4 & 5 & 6 \\
\hline \multirow{2}{*}{175} & maximum queue & 37 & 38 & 80 & 78 & 39 & 32 \\
\hline & travel time & 74. & 60. & 64. & 145. & 59. & 53. \\
\hline \multirow{2}{*}{200} & maximum queue & 38 & 39 & 79 & 79 & 41 & 34 \\
\hline & travel time & 64 & 53. & 56. & 199. & 112 & 54. \\
\hline \multirow{2}{*}{225} & maximum queue & 37 & 37 & 51 & 46 & 41 & 33 \\
\hline & travel time & 39. & 64. & 70. & 81.3 & 74. & 77. \\
\hline \multirow{2}{*}{250} & maximum queue & 45 & 38 & 51 & 54 & 46 & 44 \\
\hline & travel time & 39. & 55. & 65 & 78.2 & 74. & 89. \\
\hline \multirow{2}{*}{275} & maximum queue & 32 & 39 & 58 & 53 & 47 & 42 \\
\hline & travel time & 54. & 59. & 84. & 105. & 82. & 85. \\
\hline \multirow{2}{*}{300} & maximum queue & 36 & 39 & 57 & 52 & 31 & 37 \\
\hline & travel time & 46. & 62. & 63. & 79.5 & 57. & 67. \\
\hline
\end{tabular}

TABLE II Variation Analysis of Maximum Queue Length by Maximum Look-Ahead Distance

\begin{tabular}{|c|c|c|c|c|c|c|}
\hline $\begin{array}{c}\text { Variation } \\
\text { Sources }\end{array}$ & $S S$ & $d f$ & $M S$ & $F$ & $P$-value & $F$-crit \\
\hline Intergroup & 584 & 5 & 116.7778 & 0.578904 & 0.715738 & 2.533555 \\
\hline Withingroup & 6052 & 30 & 201.7222 & & & \\
\hline Total & 6636 & 35 & & & & \\
\hline
\end{tabular}

TABLE III Variation Analysis of Travel Time by Maximum Look-Ahead Distance

\begin{tabular}{|c|c|c|c|c|c|c|}
\hline $\begin{array}{c}\text { Variation } \\
\text { Sources }\end{array}$ & $S S$ & $d f$ & $M S$ & $F$ & $P$-value & $F$-crit \\
\hline Intergroup & 2929 & 5 & 585.7218 & 0.629552 & 0.678569 & 2.533555 \\
\hline Withingroup & 27911 & 30 & 930.3786 & & & \\
\hline Total & 30840 & 35 & & & & \\
\hline
\end{tabular}

Using the maximum queue length and travel time in TABLE I to perform a variance analysis, see TABLE II and TABLE III. In the two tables, $S S$ is the sum of squares, $d f$ is the degree of freedom, $M S$ is the mean square deviation, $F$ is the test statistics, $P$-value is the probability value under the appropriate $F, F$-crit is the critical value of $F(5,30)$ distribution under 5\% significance level. Through the variance analysis, the test statistics value for maximum queue length is less than $F$-crit, and the test statistics value for travel time is also less than $F$-crit, which means maximum look-ahead distance have no significant impact on these two evaluation indexes.

For the four model parameters including maximum lookahead distance, waiting time before diffusion, minimum headway, accepted deceleration, the test statistic values for maximum queue length and travel time are less than F-crit, which means the four model parameters have not significant impacts on maximum queue length index and travel time index. We select average standstill distance, additive part of safety distance, multiple part of safety distance and maximum deceleration as the calibrated parameters. Then we think about the calibration process based on the genetic algorithm.

\section{The calibration process for model parameters of VISSIM based on genetic algorithm}

According to the principle of genetic algorithm, we build a calibration process for calibrated parameters as Fig. 1 below:

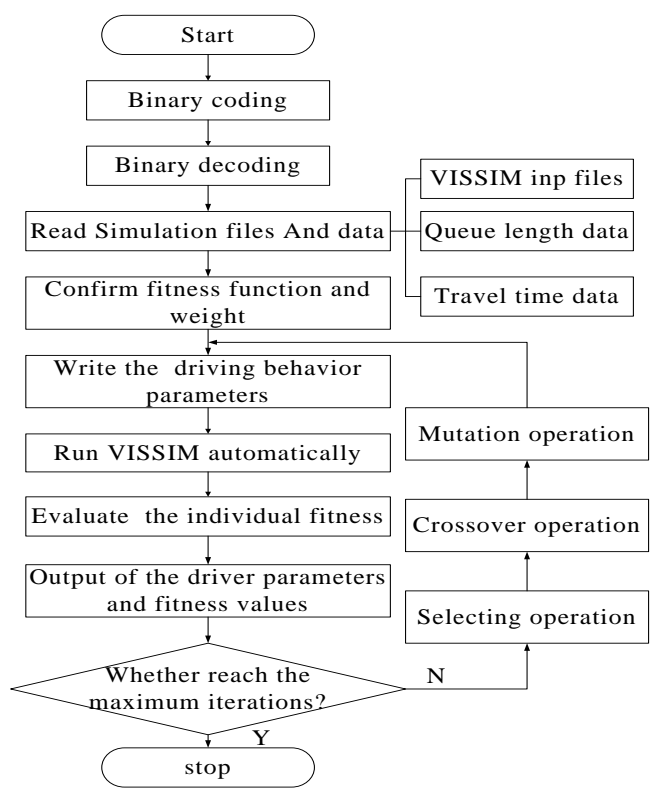

Fig. 1 Calibration process for calibrated parameters of VISSIM 


\section{The design of calibration method for VISSIM calibrated parameters based on genetic algorithm}

Combining the principle of genetic algorithm with the characteristics of VISSIM, we gain the calibration method for VISSIM calibrated parameters based on genetic algorithm, including the selection of the evaluation indexes, the encoding and decoding method of chromosomes, the determination of fitness function, the selection of operators and so on.

\section{A. Select the Evaluation Indexes}

The purpose of parameter calibration in VISSIM is to make the simulation output as close as possible to the measured value. We select maximum queue length and travel time of each approach as the evaluation indexes to prove the validity of this model. Maximum queue length and travel time can more fully reflect the characteristics of the intersection.

\section{B. The Encoding and Decoding Method of Chromosome}

This article use binary strings which consist of the digits 0 and 1 to encode the calibrated parameters, and the length of binary string is related to the required accuracy. The accuracy of coding $\delta$ can be obtained using (1 ${ }^{[11]}$. In this equation, $U_{\max }$ represents the maximum value of the calibrated parameter, $U_{\min }$ represents the minimum value of the calibrated parameter and $l$ represents the length of the string.

$$
\delta=\frac{U_{\max }-U_{\min }}{2^{l}-1},
$$

The corresponding decoding equation is shown in $(2)^{[11]}$. In this equation, $x_{i}$ represents the ith calibrated parameter $(\mathrm{i}=1,2,3,4) ; \mu_{i}$ represents the minimum value of the ith calibrated parameter; $\delta_{i}$ represents the coding accuracy of the ith calibrated parameter; $\alpha=\left(a_{1}, a_{2}, a_{3} \cdots, a_{l_{i}}\right), \alpha$ is gene expression with the vector method, and equals 0 or 1. $\beta=\left(2^{l_{i}-1}, \ldots, 4,2,1\right)^{T}$.

$$
x_{i}=\mu_{i}+\delta_{i} * \alpha * \beta,
$$

The length of the binary string and the accuracy of coding involved in this article can be received by TABLE IV, and the length of the parameter set is 15 . In this article, the number of initial populations is 10 and initial populations are generated by random numbers. In this table, $\alpha$ represents the average standstill distance; $\omega$ represents the additive part of safety distance; $\sigma$ represents the multiple part of safety distance; $\tau$ represents the maximum deceleration.

TABLE IV The Length of Binary String and the Coding Accuracy for Calibrated Parameters

\begin{tabular}{|c|c|c|c|c|c|}
\hline Parameters & Defult & Maximum & Minimum & Length & Accuracy \\
\hline$\alpha(\mathrm{m})$ & 2 & 4 & 1 & 4 & 0.2 \\
\hline$\omega$ & 2 & 5 & 0.5 & 4 & 0.3 \\
\hline$\sigma$ & 3 & 6 & 1 & 4 & 0.33 \\
\hline$\tau\left(\mathrm{m} / \mathrm{s}^{2}\right)$ & 4 & 8 & 3 & 3 & 0.71 \\
\hline
\end{tabular}

\section{The Determination of Fitness Function}

This article use the minimum tatal fitness as the solving condition. Set $F$ as the total fitness, and $x_{1}, x_{2}, x_{3}, x_{4}$ respectively represent the following parameters: average standstill distance, additive part of safety distance, multiple part of safety distance and maximum deceleration. The total fitness function is expressed by (3):

$$
F_{\text {total }}=f\left(x_{1}, x_{2}, x_{3}, x_{4}\right),
$$

Using the sum of squared error to build the fitness function, and the sum of squared error is expressed by (4). In this equation, while $i$ equals 1 , it represents the calibration accuracy index for queue length, while $i$ equals 2 , it represents the calibration accuracy index for travel time; $j$ represents the number of time bucket in data collection; $a_{i j}^{s}$ represents the model detector data of queue length or travel time in jth time bucket; $a_{i j}^{o}$ represents the collected field investigation data of queue length or travel time in jth time bucket.

$$
F_{i}=\frac{\sum_{j}\left(a_{i j}^{s}-a_{i j}^{o}\right)^{2}}{a_{i j}^{o}},
$$

Using weight coefficient transformation method to determine the total fitness function in the condition of calibrating the queue length and travel time at the same time. $\alpha$ represents weight coefficient in (5). While $\alpha$ equals 0.5 , it means the calibration accuracy index of queue length and the calibration accuracy index of travel time are equally important; while $\alpha$ is greater than 0.5 , it means the calibration accuracy index of queue length is more important.

$$
F_{\text {total }}=a F_{1}+(1-a) F_{2},
$$

\section{Genetic Operators}

1) Selection operator: According to the fitness of each individual, and the probability that each individual is selected is proportional to the individual fitness value. Assume that the population size is $I$, the individual fitness value for $i$ is $F_{i}$, the probability that the individual is selected into the next generation is $P_{i}$, and $P_{i}$ can be expressed by $(6)^{[11]}$.

$$
P_{i}=\frac{F_{i}}{\sum_{i=1}^{I} F_{i}}
$$

2) Crossover operator: Select the single-point crossover method to confirm the crossover operation, and the individuals are made pairs. Set a crossing randomly to the two paired individuals, then exchange the part chromosome of the two paired individuals in that crossing. After that, we gain two new individuals. In this article, the number of individual genes is 15 , so we have 14 possible crossings. The crossover probability ${ }^{[12]}$ in this article is defined as 0.75 . 
3) Mutation operator: Select the basic bit mutation operator to confirm the mutation operation. Use the mutation probability to determine the change-point for each individual. Invert the gene's value of the change point, then we gain a new generation. The mutation probability ${ }^{[12]}$ in this article is defined as 0.05 . The number of this population genes is 150 , so we have about 8 genes to finish the mutation operation.

\section{Example Analysis}

Select the intersection of Ronghua South Road and Rongjing East Street in Yizhuang Economic and Technological Development Zone of Beijing as the experimental region. The geometric condition of this intersection is as follows: the northeast approach has a exclusive right-turn lane, a exclusive straight lane, a lane shared by left turning and straight ahead traffic and a nonmotor vehicle lane; the geometric condition of the southwest approach is consistent with the northeast approach; the southeast approach has a exclusive left-turn lane, three exclusive straight lane, a lane shared by right turning and straight ahead traffic and a non-motor vehicle lane; the geometric condition of the northwest approach is consistent with the southeast approach. The traffic flow data came from the video collection from 5:00 pm to $6: 00 \mathrm{pm}$ on July 13th, 2012. The simulation model of the experimental region is shown below in Fig. 2:

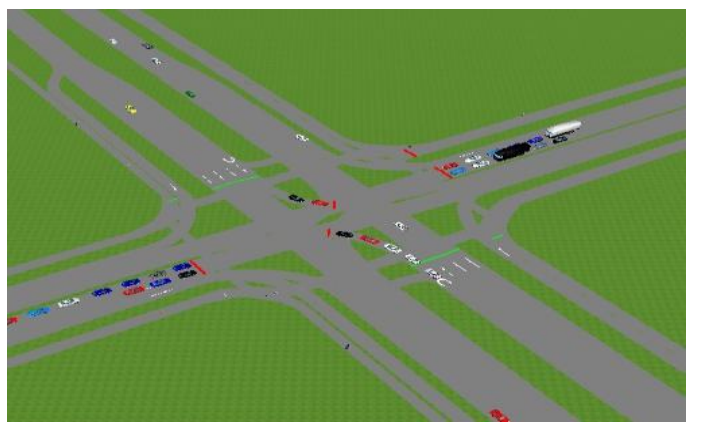

Fig. 2 The intersection of Ronghua South Road and Rongjing East Street

\section{A. Equal Weight Coefficient}

When the weight coefficient equals 0.5 , which means the queue length and travel time are equally weighted. The values of driving behavior parameters after calibration are shown in TABLE $\mathrm{V}$, and the letters in this table has been defined above:

TABLE V The Values of Default and Optimal Values by Equal Weight Coefficient

\begin{tabular}{|c|c|c|}
\hline Parameters & Defult Values & Values after Calibration \\
\hline$\alpha(\mathrm{m})$ & 2 & 2.4 \\
\hline$\omega$ & 2 & 1.1 \\
\hline$\sigma$ & 3 & 3 \\
\hline$\tau\left(\mathrm{m} / \mathrm{s}^{2}\right)$ & -4 & -6.57 \\
\hline
\end{tabular}

Using the fitness function defined in Chapter 5 to analyze the convergence performance of the fitness average and optimal values in generations under equal weight coefficient as Fig. 3:

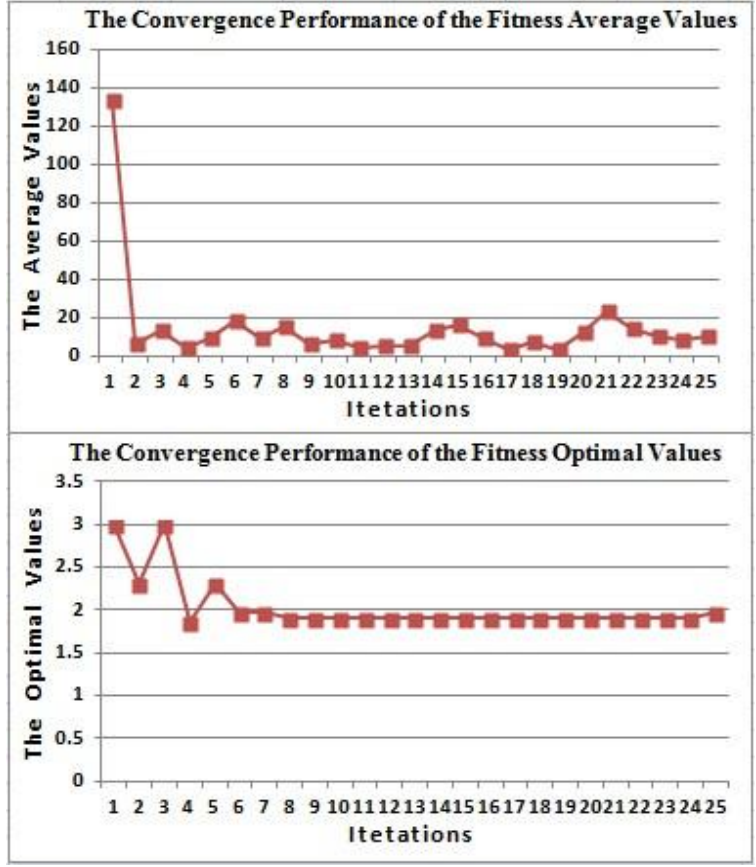

Fig. 3 Convergence performance of the fitness average and optimal values in generations by equal weight coefficient

Making a comparison among the average value of the real measured maximum queue length (travel time), the average value of the maximum queue length (travel time) when the calibrated parameters get the default values and the average value of the maximum queue length (travel time) after the parameters are calibrated in a simulation hour. The results are shown in Fig. 4:

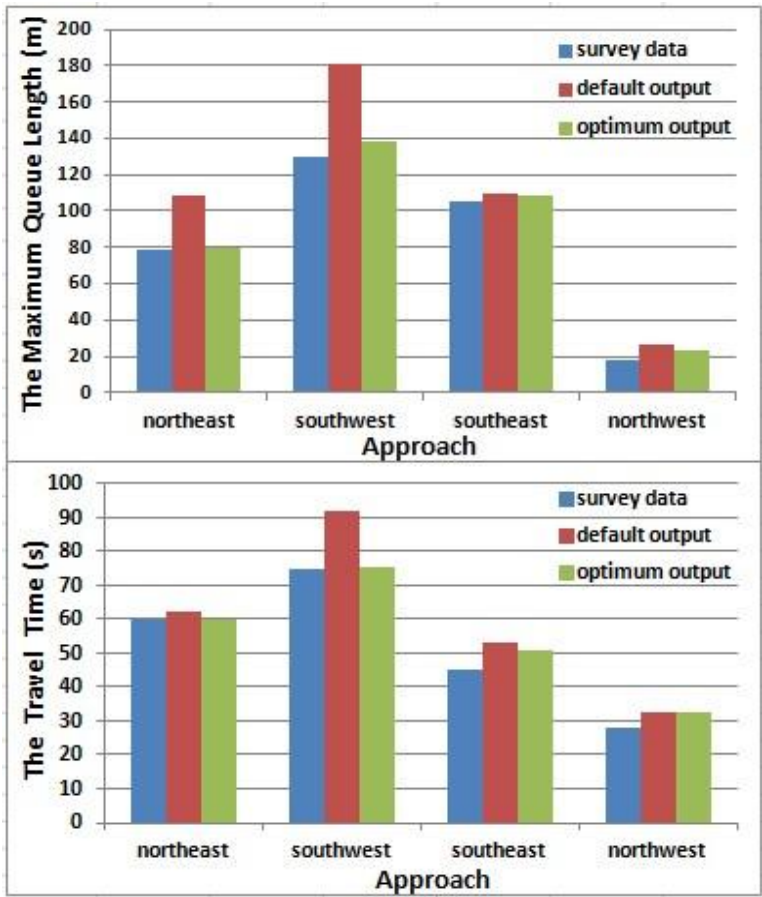

Fig. 4 Comparison of the maximum queue length and travel time by equal weight coefficient 
Using the mean absolute relative error as the total error, and the mean absolute relative error of maximum queue length and travel time can be expressed by (7):

$$
\operatorname{MARE}_{i j}=\frac{1}{4} \sum_{j=1}^{4}\left|\frac{\theta_{i j}^{s}-\theta_{i j}^{o}}{\theta_{i j}^{o}}\right|,
$$

In this equation, while $i$ equals $1, \theta_{i j}^{s}$ and $\theta_{i j}^{o}$ correspond to the simulation output maximum queue length and the real measured maximum queue length for each approach, and the unit is meter; While $i$ equals $2, \theta_{i j}^{s}$ and $\theta_{i j}^{o}$ correspond to the simulation output travel time and the real measured travel time for each approach, and the unit is second.

For the maximum queue length index, $M A R E_{1 j}$ is 0.313 before calibration, and $M A R E_{1 j}$ is 0.098 after calibration. The mean absolute relative error of maximum queue length for each approach decreases by 0.215 and the improvement rate is $68.7 \%$, which means the improvement of maximum queue length is obvious.

For the travel time index, $M A R E_{2 j}$ is 0.152 before calibration, and $M A R E_{2 j}$ is 0.073 after calibration. The mean absolute relative error of travel time for each approach decreases by 0.079 . The mean absolute relative error of travel time has been less than 0.15 before calibration, and the improvement of travel time is not obvious.

\section{B. Unequal Weight Coefficients}

It can be obtained from the above article that the mean absolute relative error of travel time has been less than 0.15 before calibration and the mean absolute relative error of the maximum queue length has been more than 0.30 before calibration. We need to focus on the calibration of maximum queue length when we calibrate the maximum queue length and the travel time at the same time. Change the weight coefficient of maximum queue length from 0.5 to 0.8 to continue the calibration of this case and get the results of driving behavior parameters after calibration. Shown as TABLE VI, and the letters in this table has been defined above:

TABLE VI The Values of Default and Optimal Values by Equal and Unequal Weight Coefficients

\begin{tabular}{|c|c|c|c|}
\hline Parameters & $\begin{array}{c}\text { Defult } \\
\text { Values }\end{array}$ & $\begin{array}{c}\text { Values after Calibration } \\
\text { (weight is } 0.5 \text { ) }\end{array}$ & $\begin{array}{c}\text { Values after Calibration } \\
\text { (weight is 0.8 ) }\end{array}$ \\
\hline$\alpha(\mathrm{m})$ & 2 & 2.4 & 2 \\
\hline$\omega$ & 2 & 1.1 & 2.6 \\
\hline$\sigma$ & 3 & 3 & 1.67 \\
\hline$\tau\left(\mathrm{m} / \mathrm{s}^{2}\right)$ & -4 & -6.57 & -6.57 \\
\hline
\end{tabular}

Using the fitness function defined in Chapter 5 to analyze the convergence performance of the fitness average and optimal values in generations under equal and unequal weight coefficients as Fig. 5:
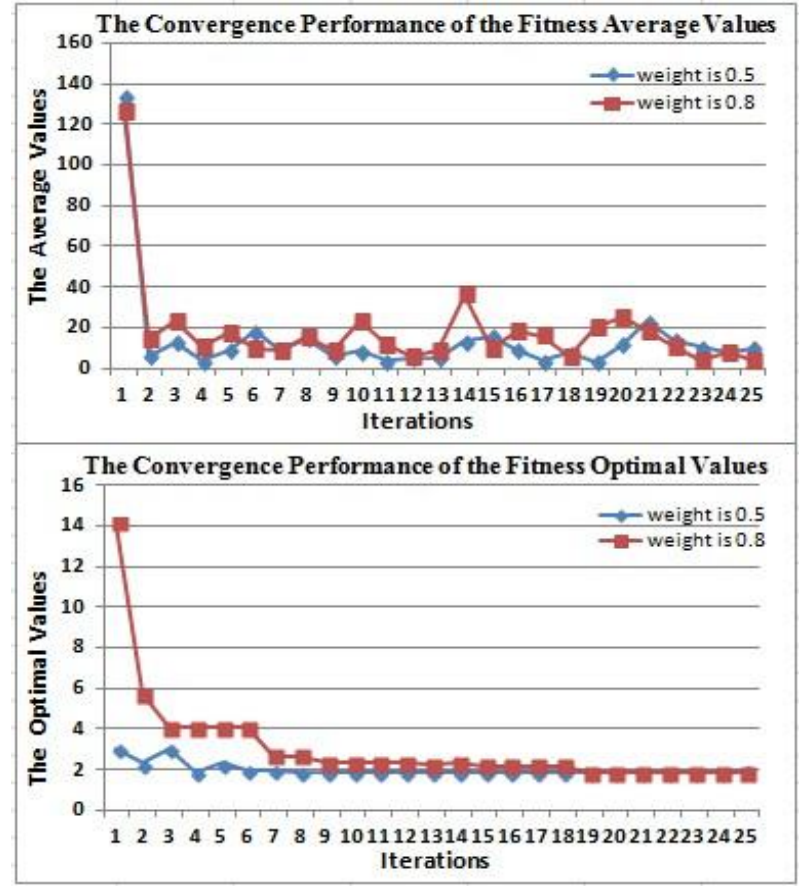

Fig. 5 Convergence performance of the fitness average and optimal values in generations by equal and unequal weight coefficients

Making a comparison among the average value of the real measured maximum queue length (travel time), the average value of the maximum queue length (travel time) when the calibrated parameters get the default values and the average value of the maximum queue length (travel time) after the parameters are calibrated in a simulation hour. The results are shown in Fig. 6:

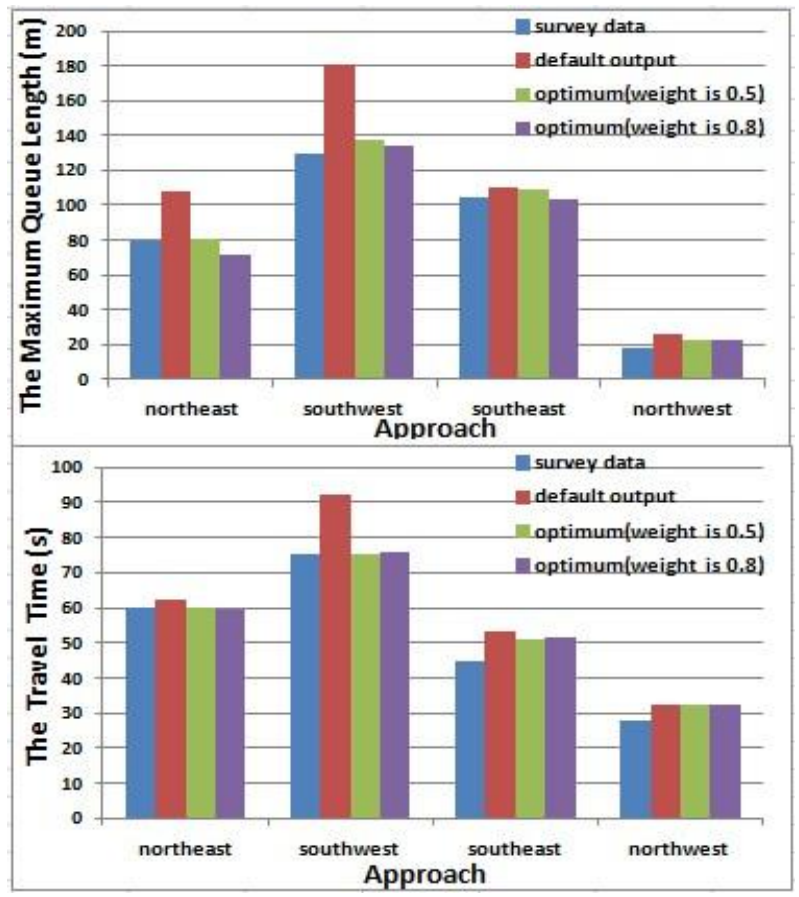

Fig. 6 Comparison of the maximum queue length and travel time by equal and unequal weight coefficients 
Using the mean absolute relative error as the total error to analyze the impact of weight coefficient on the maximum queue length and travel time: when the weight coefficient equals 0.5 , the mean absolute relative error value of maximum queue length $\left(M A R E_{1 j}\right)$ is 0.098 , and the mean absolute relative error value of travel time $\left(M A R E_{2 j}\right)$ is 0.073 . When the weight coefficient is adjusted to 0.8 , the mean absolute relative error value of maximum queue length $\left(M A R E_{1 j}\right)$ is 0.093, and the mean absolute relative error value of travel time $\left(M A R E_{2 j}\right)$ is 0.080 . In this case, after increasing the weight coefficient of maximum queue length, the error of maximum queue length under the optimal parameters shows some improvement.

For getting a more comprehensive analysis on the impact of weight coefficient, we analyze the output results when the weight is changed to a minimum value of 0 and a maximum value of 1 . Using the above method to calibrate this case and using the mean absolute relative error to analyze the impact of weight coefficient on the maximum queue length and travel time respectively. The results are shown in Fig. 7:

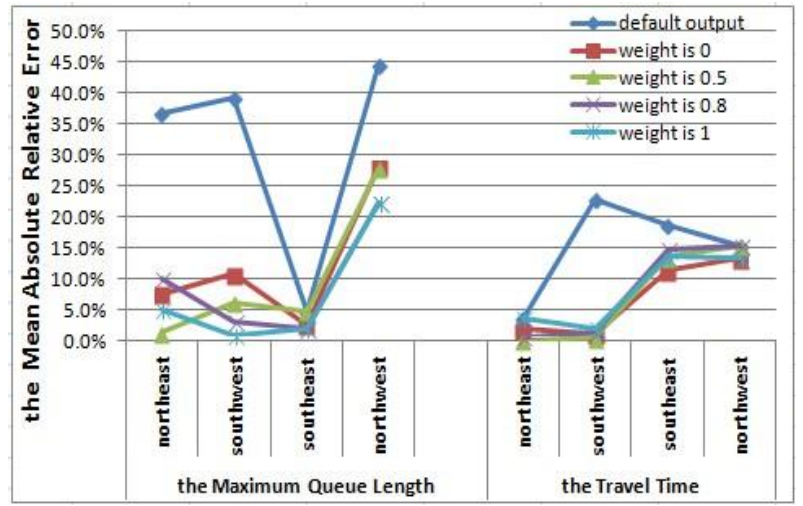

Fig.7 Comparison of evaluation indexes by four different weight coefficients

From the above figure, while the weight coefficient equals 0 , the mean absolute relative error of maximum queue length is greater than the errors of maximum queue lengths under other weight coefficients, and the mean absolute relative error of travel time is relatively low. While the weight coefficient equals 1 , the mean absolute relative error of maximum queue length is the lowest one and the mean absolute relative error of maximum queue length is the highest one. When the weight coefficients are 0.5 and 0.8 , the variation trends of these two curves basically conform to the definition of fitness function, see (5). According to the described calibration method in this article, the weight coefficient should be confirmed by the practical situation of the researched intersection. After we balance the impact of maximum queue length index against the impact of travel time index, we can confirm a fine weight coefficient to complete the parameter calibration of this model.

\section{Conclusion}

This article studied the parameter calibration method of VISSIM simulation model, and put forward a calibration process for VISSIM based on genetic algorithm, and proved the practicality and effectiveness of this calibration method with an example. The errors of simulation results were within the acceptable limits, and this method truly reproduced the running condition and provided a appropriate platform for the formulation of later optimization scheme.

\section{Acknowledgment}

Prof. Yong Qin put forward a lot of valuable opinions on my study, Ph. D. Yanfang Yang and Qinghua Zhang helped me a lot in the process of this article, I express my sincere thanks to them.

\section{References}

[1] R. F. Benekohal, " Procedure for validation of microscopic traffic flow simulation models," 70 th Transportation Research Board Annual Meeting, Washington D.C, USA, 1991.

[2] B. R. Hellinga, " Requirements for the calibration of traffic simulation models," Canadian Society of Civil Engineering, Ontario, CA, 1998.

[3] R. L. Cheu, et al, "Calibration of Fresim for a Singapore's expressway using genetic algorithm," Journal of Transportation Engineering, vol. 124, pp. 526-535, 1998.

[4] B. Park and J. D. Schneeberger, " Microscopic simulation model calibrati-on and validation: case study of VISSIM simulation model for a coordinated actuated signal system," Transportation Research Record, no. 1856, pp.185-192, 2003.

[5] B. Park and H. Qi, " Development and evaluation of simulation model calibration procedure," $84^{\text {st }}$ Transportation Research Board Annual Meeting, Washington D.C, USA, 2005.

[6] Z. M. Li and X.Y.Yan, " Study on correction method of traffic simulation model based on genetic algorithm," Communications Standardization, iss. 4, pp. 21-23, 2006.

[7] Y.Wang, Study on the relationship between the access of urban express-way and bus station based on micro-simulaition, Beijing, China: Beijing University of Technology, 2010.

[8] Q. Yu, M. Wang and X. H. Deng, " Simulation parameter calibration of single signalized intersection based on orthogonal experiment method," Journal of Highway and Transportation Research and Development, vol. 29, pp. 57-63, 2012.

[9] Y. X. Liu, The analysis and modeling of the capacity of the expressway resource allocation with the bus lane based on microscopic simulation, Beijing, China: Beijing Jiaotong University, 2009.

[10] N. Kishorjit and B. Sivaji, "Genetic algorithm in feature selection for CRF based manipuri multiword expression identification," International Journal of Computer Science \& Information Technology, vol. 3, 2011.

[11] X. P. Wang and L. M. Cao, Genetic algorithms- theory, application and software implementation, Xian: Jiaoda Press, 2002: 24-39.

[12] A. Q. Wang and W. Zeng, "Two traffic volume forecast methods based on genetic algorithms," Communications Standardization, iss. 5, pp. 60$62,2004$. 